\title{
Efficient Heterogeneous Asymmetric Transfer Hydrogenation of Ketones Using Highly Recyclable and Accessible Silica-Immobilized Ru-TsDPEN Catalysts
}

\author{
Pei Nian Liu, Pei Ming Gu, Fei Wang and Yong Qiang Tu* \\ Department of Chemistry and State Key Laboratory of Applied Organic Chemistry \\ Lanzhou University, Lanzhou 730000, P. R. China. \\ tuyq@lzu.edu.cn
}

\section{Supporting Information}

1. General.

2. Synthesis of $(1 R, 2 R)-4$.

3. General procedure for the synthesis of immobilized ligands 5-7.

4. Synthesis of the modified ligand 8.

5. General procedure for the transfer hydrogenation of ketones using the immobilized catalysts.

6. Determination of the leaching of $\mathrm{Ru}$ from the catalyst $\mathrm{Ru}-5$ in the recycling reductions of acetophenone.

7. Analytical data for obtained chiral aromatic alcohols.

8. Reference. 


\section{General:}

Fomic acid-triethylamine azeotrope was prepared by adding triethylamine to fomic acid (ratio: 2:5) and then distilled under reduced pressure. Acetophenone was distilled from $\mathrm{KMnO}_{4}$ and $\mathrm{CH}_{2} \mathrm{Cl}_{2}$ was distilled from $\mathrm{CaH}_{2}$. Triethylamine was distilled and stored avoiding light. $\left[\mathrm{RuCl}_{2}(p \text {-cymene) }]_{2}\right.$ was prepared according to literature ${ }^{1}$ and $(1 R, 2 R)$-TsDPEN was prepared by standard method. Amorphous silica gel (60-100 mesh), mesoporous silicas of MCM-41 and SBA-15 were heated at $150{ }^{\circ} \mathrm{C}$ for $3 \mathrm{~h}$ and cooled under argon before use. Toluene was freshly distilled from a deep-blue solution of sodium-benzophenone under argon. Other reagents and chemicals were used as received.

${ }^{1} \mathrm{H}$ NMR and ${ }^{13} \mathrm{C}$ NMR spectra were recorded on Varian Mercury-plus $300 \mathrm{BB}$ with TMS as internal standard. HRMS data were measured with ESI techniques (Bruker Apex II). Elemental analysis was performed on elementar vario EL and FT-IR spectra was performed on Nicolet NEXUS 670. The pore sizes and surface areas were determined on Micromeritics ASAP 2010 Accelerated Surface Area and Porosimetry System. The inductively coupled plasma atomic emission spectrometry (ICP-AES) was performed on Perkin-Elmer ICP/6500 inductively coupled plasma spectrometer. Optical rotation values were measured on Perkin-Elmer 341 polarimeter. Enantiomeric excess values were determined by GC with a CP-Chirasil-DEX CB column $(25 \mathrm{~m} \times$ $0.32 \mathrm{~mm}$ ) on Varian CP-3800 or by HPLC with Chiralcel OB or OJ column on Agilent 1100 Series.

\section{Synthesis of $(1 R, 2 R)-4$ :}

$(1 R, 2 R)$-DPEN $2(0.636 \mathrm{~g}, 3.0 \mathrm{mmol})$ and triethylamine $(0.6 \mathrm{~mL})$ were dissolved in $\mathrm{CH}_{2} \mathrm{Cl}_{2}(30 \mathrm{~mL})$, and 2-(4-chlorosulfonylphenyl)ethyltrimethoxysilane $3(0.75 \mathrm{~g}, 2.31 \mathrm{mmol})$ in $\mathrm{CH}_{2} \mathrm{Cl}_{2}(30 \mathrm{~mL})$ was 
then added dropwise at $0{ }^{\circ} \mathrm{C}$. The reaction mixture was then allowed to warm to room temperature slowly and stirred for $3 \mathrm{~h}$. After the solvent was removed in vacuo, the residue was fast passed through a short column (silica gel, eluent: $\mathrm{Et}_{3} \mathrm{~N}: \mathrm{CH}_{3} \mathrm{OH}: \mathrm{CH}_{2} \mathrm{Cl}_{2}=1: 10: 100$ ) and concentrated in vacuo to afford $(R, R)-\mathbf{4}$ as beige glass.

Yield: $86 \%$; $[\alpha]_{\mathrm{D}}{ }^{20}-36\left(c 0.8, \mathrm{CH}_{2} \mathrm{Cl}_{2}\right) ;{ }^{1} \mathrm{H}$ NMR $\left(300 \mathrm{MHz}, \mathrm{CDCl}_{3}\right) \quad \delta \quad 0.89-0.95(\mathrm{~m}, 2 \mathrm{H}), 1.50(\mathrm{~s}$, 2H, $\left.\mathrm{NH}_{2}\right), 2.65-2.70(\mathrm{~m}, 2 \mathrm{H}), 3.59(\mathrm{~s}, 9 \mathrm{H}), 4.12(\mathrm{~d}, J=5.4 \mathrm{~Hz}, 1 \mathrm{H}), 4.37(\mathrm{~d}, J=5.4 \mathrm{~Hz}, 1 \mathrm{H})$, 6.99-7.34 (m, 14H) ${ }^{13} \mathrm{C}$ NMR $\left(75 \mathrm{MHz}, \mathrm{CDCl}_{3}\right) \quad \delta 11.0,28.5,50.6,60.4,63.2,126.5,126.9$, 127.3, 127.4, 127.8, 128.2, 128.3, 137.3, 139.1, 141.3, 148.8; IR 3352, 3296, 3171, 3060, 3028, $2941,2841,1902,1691,1600,1495,1454,1410,1343,1322,1265,1191,1157,1088,1013 \mathrm{~cm}^{-1}$ ESI-HRMS, calcd for $\mathrm{C}_{25} \mathrm{H}_{32} \mathrm{~N}_{2} \mathrm{O}_{5} \mathrm{SSi}+\mathrm{H}^{+}$501.1874, found 501.1881.

\section{General procedure for the synthesis of immobilized ligands 5-7:}

The predried silica $(3 \mathrm{~g})$ and ligand $(R, R)-4(0.25 \mathrm{~g})$ were added to dry toluene $(40 \mathrm{~mL})$ and the mixture was refluxed for $24 \mathrm{~h}$ under argon. After filtration, the white solid was washed by toluene (60 mL), mixture solvent of $\mathrm{CH}_{2} \mathrm{Cl}_{2}$ and $\mathrm{CH}_{3} \mathrm{OH}(1: 1,100 \mathrm{~mL})$. Then the solid was suspended in the mixture solvent $\left(\mathrm{CH}_{2} \mathrm{Cl}_{2}: \mathrm{CH}_{3} \mathrm{OH}=1: 1,50 \mathrm{~mL}\right)$ and stirred overnight. After filtration and thorough wash with the mixture solvent $\left(\mathrm{CH}_{2} \mathrm{Cl}_{2}: \mathrm{CH}_{3} \mathrm{OH}=1: 1,50 \mathrm{~mL}\right)$ and $\mathrm{CH}_{3} \mathrm{OH}(50 \mathrm{ml})$, the solid was dried at $60{ }^{\circ} \mathrm{C}$ in vacuo for $24 \mathrm{~h}$ to give the immobilized ligand.

Ligand 5: IR 3440, 2960, 1642, 1509, 1464, 1097, 801, 702, 559, $469 \mathrm{~cm}^{-1}$; elemental analysis: $\mathrm{N}$ 0.41, C 4.71, S 0.48, H 0.59; average pore size: $90.2 \AA$; $\mathrm{S}_{\mathrm{BET}}: 324$ m²/g. 
Ligand 6: IR 3442, 2962, 2860, 1627, 1461, 1081, 812, 698, $455 \mathrm{~cm}^{-1}$; elemental analysis: N 0.38, C 6.52, S 0.47, H 1.04; pore size: $18.7 \AA$; $\mathrm{S}_{\mathrm{BET}}: 1150 \mathrm{~m}^{2} / \mathrm{g}$.

Ligand 7: IR 3438, 2958, 2856, 1635, 1462, 1084, 811, 701, $462 \mathrm{~cm}^{-1}$; elemental analysis: N 0.28, C 5.74, S 0.31, H 0.54; pore size: $61.8 \AA$; $\mathrm{S}_{\mathrm{BET}}: 497 \mathrm{~m}^{2} / \mathrm{g}$.

\section{Synthesis of the modified ligand 8:}

Immobilized ligand $5(0.3 \mathrm{~g})$ was added to hexamethyldisiloxane (HMDSO, $10 \mathrm{~mL})$ and the mixture was refluxed for $18 \mathrm{~h}$ under argon. After filtration and thorough wash with $\mathrm{CH}_{3} \mathrm{OH}(30 \mathrm{~mL})$, the solid was dried at $60{ }^{\circ} \mathrm{C}$ in vacuo for $24 \mathrm{~h}$ to give ligand 8 .

Ligand 8: IR 3439, 2970, 2845, 1637, 1460, 1095, 965, 802, 700, $467 \mathrm{~cm}^{-1}$; elemental analysis: N 0.33, C 5.55, S 0.41, H 1.10; average pore size: $87.4 \AA$; $\mathrm{S}_{\mathrm{BET}}: 313$ m²/g.

\section{General procedure for the transfer hydrogenation of ketones using the immobilized catalysts:}

Immobilized ligand $(0.0068 \mathrm{mmol}),\left[\mathrm{RuCl}_{2}(p \text {-cymene })\right]_{2}(0.002 \mathrm{mmol})$ and triethylamine $(0.016$ mmol) were stirred in dry $\mathrm{CH}_{2} \mathrm{Cl}_{2}(0.4 \mathrm{~mL})$ for $1 \mathrm{~h}$ under argon at $28{ }^{\circ} \mathrm{C}$. Then the formic acid-triethylamine azeotrope $(0.2 \mathrm{~mL})$ and the ketone $(0.4 \mathrm{mmol})$ were added and the mixture was stirred at $40{ }^{\circ} \mathrm{C}$ and monitored by TLC. After the completion of the reaction, dry $\mathrm{CH}_{2} \mathrm{Cl}_{2}(1 \mathrm{~mL})$ was added and the mixture was stirred for $1 \mathrm{~min}$, then the reactor was centrifuged $(2000 \mathrm{r} / \mathrm{min})$ for 1-2 min and the solution was removed with syringe. The catalyst was then washed with $\mathrm{CH}_{2} \mathrm{Cl}_{2}(1$ $\mathrm{mL}$ ) twice and $\mathrm{CH}_{2} \mathrm{Cl}_{2}$ was removed; a new reaction could be conducted by adding formic 
acid-triethylamine azeotrope $(0.2 \mathrm{~mL})$ and ketone $(0.4 \mathrm{mmol})$ in turn to the recovered catalyst. The solution containing the product was fast passed through a short column (silica gel, eluent: $\mathrm{Et}_{2} \mathrm{O}$ ) and the conversion and the ee value were then determined by chiral GC on CP-Chirasil-DEX CB column $(25 \mathrm{~m} \times 0.32 \mathrm{~mm})$ or by HPLC with Chiralcel OB or OJ column on Agilent 1100 Series.

\section{Determination of the leaching of $\mathrm{Ru}$ from the catalyst $\mathrm{Ru}-5$ in the recycling reductions of acetophenone.}

The predried recovered catalyst Ru-5 $(50.0 \mathrm{mg})$ with the powder mixture of $\mathrm{KOH}(1.0 \mathrm{~g})$ and $\mathrm{KNO}_{3}$ (0.2 g) was heated to $650{ }^{\circ} \mathrm{C}$ for 5-10 min, then cooled, dissolved in hot water and added $12 \mathrm{M} \mathrm{HCl}$ $(4 \mathrm{~mL})$ to acidify the solution, then the solution was diluted to $50.0 \mathrm{~mL}$ and the amount of metal $\mathrm{Ru}$ was determined by ICP-AES.

The leached $\mathrm{Ru}$ in organic solutions after the reaction was dissolved in $0.3 \mathrm{M} \mathrm{HCl} \mathrm{(10} \mathrm{mL)} \mathrm{and}$ determined by ICP-AES.

$\mathbf{R u} / \mathrm{SiO}_{2}$ (weight): before use: $0.54 \%$, after 5 uses: $0.35 \%$.

Amount of leached Ru in organic solutions: after the 1st run: $11 \mu \mathrm{g}$, after the 2 nd run: $15 \mu \mathrm{g}$, after the 3rd run: $17 \mu \mathrm{g}$, after the 4th run: $24 \mu \mathrm{g}$, after the 5th run: $34 \mu \mathrm{g}$.

\section{Analytical data for obtained chiral aromatic alcohols:}<smiles>CCCCCO</smiles> 
$[\alpha]_{\mathrm{D}}{ }^{28}+52\left(c 1.1, \mathrm{CHCl}_{3}\right) ;{ }^{1} \mathrm{H}$ NMR $\left(300 \mathrm{MHz}, \mathrm{CDCl}_{3}, 97 \%\right.$ ee $) \quad \delta \quad 1.45(\mathrm{~d}, J=6.6 \mathrm{~Hz}, 3 \mathrm{H}), 2.29$ (s, $1 \mathrm{H}, \mathrm{OH}), 4.80-4.86(\mathrm{~m}, 1 \mathrm{H}), 7.23-7.35(\mathrm{~m}, 5 \mathrm{H}) ;{ }^{13} \mathrm{C} \mathrm{NMR}\left(75 \mathrm{MHz}, \mathrm{CDCl}_{3}\right) \quad \delta$ 25.0, 70.2, 125.3, 127.3, 128.4, 145.8; GC analysis: CP-Chirasil-DEX CB column $(25 \mathrm{~m} \times 0.32 \mathrm{~mm})$, column temprature $=126{ }^{\circ} \mathrm{C}$, (isothemal), injector temprature $=250{ }^{\circ} \mathrm{C}$, detector temperature (FID) $=300$, carrier gas: $\mathrm{N}_{2}$, inlet pressure $=7 \mathrm{psi} ; \mathrm{t}_{\mathrm{R}}=8.1 \mathrm{~min}, \mathrm{t}_{\mathrm{S}}=8.8 \mathrm{~min}$.

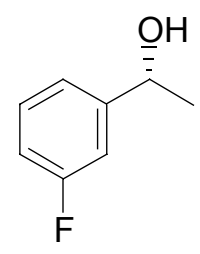

$\mathrm{C}_{8} \mathrm{H}_{9} \mathrm{FO}, \mathrm{FW}=140.15$

$[\alpha]_{\mathrm{D}}{ }^{28}+36\left(c 1.8, \mathrm{CHCl}_{3}, 94 \%\right.$ ee); ${ }^{1} \mathrm{H} \mathrm{NMR}\left(300 \mathrm{MHz}, \mathrm{CDCl}_{3}\right) \quad \delta 1.42-1.45(\mathrm{~m}, 3 \mathrm{H}), 2.69(\mathrm{~s}, 1 \mathrm{H}$, $\mathrm{OH}), 4.78-4.85(\mathrm{~m}, 1 \mathrm{H}), 6.89-6.96(\mathrm{~m}, 1 \mathrm{H}), 7.03-7.09(\mathrm{~m}, 2 \mathrm{H}), 7.23-7.31(\mathrm{~m}, 1 \mathrm{H}) ;{ }^{13} \mathrm{C}$ NMR $(75$ $\left.\mathrm{MHz}, \mathrm{CDCl}_{3}\right) \delta 25.1,69.6,112.1,112.4,113.9,114.2,120.9,129.8,129.9,148.4,148.5,161.3$, 164.5; GC analysis: CP-Chirasil-DEX CB column $(25 \mathrm{~m} \times 0.32 \mathrm{~mm})$, column temprature $=135^{\circ} \mathrm{C}$, (isothemal), injector temprature $=250{ }^{\circ} \mathrm{C}$, detector temperature (FID) $=300$, carrier gas: $\mathrm{N}_{2}$, inlet pressure $=7 \mathrm{psi} ; \mathrm{t}_{\mathrm{R}}=8.6 \mathrm{~min}, \mathrm{t}_{\mathrm{S}}=9.6 \mathrm{~min}$

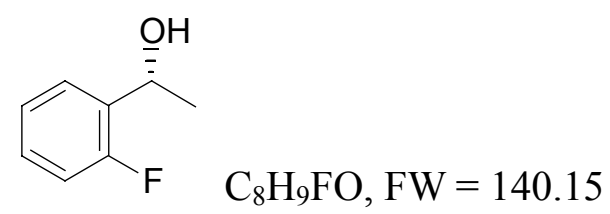

$[\alpha]_{\mathrm{D}}{ }^{28}+38\left(c 1.2, \mathrm{CHCl}_{3}, 93 \% \mathrm{ee}\right) ;{ }^{1} \mathrm{H} \mathrm{NMR}\left(300 \mathrm{MHz}, \mathrm{CDCl}_{3}\right) \quad \delta \quad 1.53(\mathrm{~d}, J=6.6 \mathrm{~Hz}, 3 \mathrm{H}), 2.20$ (s, 1H, OH), 4.96-5.03 (m, 1H), 7.43-7.47 (m, 2H), 7.75-7.80 (m, 2H); ${ }^{13} \mathrm{C}$ NMR (75 MHz, $\left.\mathrm{CDCl}_{3}\right)$ $\delta$ 25.0, 70.4, 123.8, 125.7, 126.1, 127.6, 127.9, 128.2, 132.8, 133.3, 143.2; GC analysis: CP-Chirasil-DEX CB column $(25 \mathrm{~m} \times 0.32 \mathrm{~mm})$, column temprature $=127^{\circ} \mathrm{C}$, (isothemal), injector temprature $=250{ }^{\circ} \mathrm{C}$, detector temperature (FID) $=300$, carrier gas : $\mathrm{N}_{2}$, inlet pressure $=7 \mathrm{psi} ; \mathrm{t}_{\mathrm{R}}=$ $9.7 \mathrm{~min}, \mathrm{t}_{\mathrm{S}}=10.5 \mathrm{~min}$. 


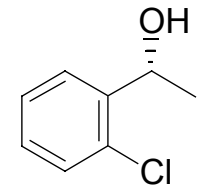

$\mathrm{C}_{8} \mathrm{H}_{9} \mathrm{ClO}, \mathrm{FW}=156.61$

$[\alpha]_{\mathrm{D}}{ }^{28}+54\left(c 2.3, \mathrm{CHCl}_{3}, 94 \%\right.$ ee) ${ }^{1} \mathrm{H} \mathrm{NMR}\left(300 \mathrm{MHz}, \mathrm{CDCl}_{3}\right) \quad \delta \quad 1.42-1.45(\mathrm{~m}, 3 \mathrm{H}), 2.78(\mathrm{~s}, 1 \mathrm{H}$, $\mathrm{OH}), 5.22-5.26(\mathrm{~m}, 1 \mathrm{H}), 7.12-7.30(\mathrm{~m}, 3 \mathrm{H}), 7.53-7.55(\mathrm{~m}, 1 \mathrm{H}) ;{ }^{13} \mathrm{C} \mathrm{NMR}\left(75 \mathrm{MHz}, \mathrm{CDCl}_{3}\right) \delta$ 23.4, 66.7, 126.3, 127.1, 128.2, 129.2, 131.4, 143.0; GC analysis: CP-Chirasil-DEX CB column (25 $\mathrm{m} \times 0.32 \mathrm{~mm})$, column temprature $=160{ }^{\circ} \mathrm{C}$, (isothemal), injector temprature $=250{ }^{\circ} \mathrm{C}$, detector temperature $(F I D)=300$, carrier gas: $\mathrm{N}_{2}$, inlet pressure $=7 \mathrm{psi} ; \mathrm{t}_{\mathrm{R}}=7.2 \mathrm{~min}, \mathrm{t}_{\mathrm{S}}=7.8 \mathrm{~min}$.

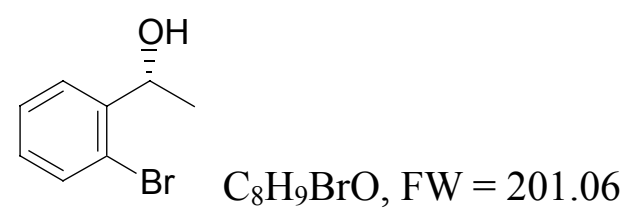

$[\alpha]_{\mathrm{D}}{ }^{28}+48\left(c 1.7, \mathrm{CHCl}_{3}, 93 \%\right.$ ee $) ;{ }^{1} \mathrm{H} \mathrm{NMR}\left(300 \mathrm{MHz}, \mathrm{CDCl}_{3}\right) \quad \delta 1.42(\mathrm{~d}, J=6.3 \mathrm{~Hz}, 3 \mathrm{H}), 2.81$

(s, 1H, OH), 5.14-5.20 (m, 1H), $7.08(\mathrm{t}, J=7.5 \mathrm{~Hz}, 1 \mathrm{H}), 7.30(\mathrm{t}, J=7.2 \mathrm{~Hz}, 1 \mathrm{H}), 7.45-7.54(\mathrm{~m}, 2 \mathrm{H})$;

${ }^{13} \mathrm{C}$ NMR $\left(75 \mathrm{MHz}, \mathrm{CDCl}_{3}\right) \quad \delta 23.5,69.0,121.5,126.6,127.7,128.6,132.5,144.6$; GC analysis: CP-Chirasil-DEX CB column $(25 \mathrm{~m} \times 0.32 \mathrm{~mm})$, column temprature $=170{ }^{\circ} \mathrm{C}$, (isothemal), injector temprature $=250{ }^{\circ} \mathrm{C}$, detector temperature $(\mathrm{FID})=300$, carrier gas: $\mathrm{N}_{2}$, inlet pressure $=7 \mathrm{psi} ; \mathrm{t}_{\mathrm{R}}=$ $6.5 \mathrm{~min}, \mathrm{t}_{\mathrm{S}}=7.3 \mathrm{~min}$.

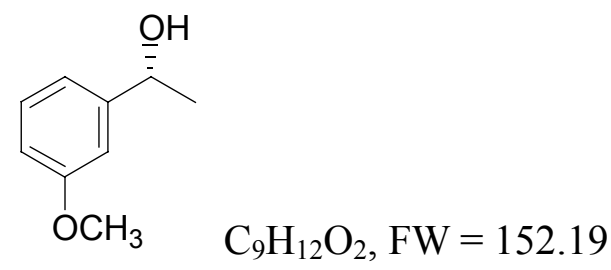

$[\alpha]_{\mathrm{D}}{ }^{28}+39\left(c 1.0, \mathrm{CHCl}_{3}, 96 \%\right.$ ee); ${ }^{1} \mathrm{H} \mathrm{NMR}\left(300 \mathrm{MHz}, \mathrm{CDCl}_{3}\right) \quad \delta 1.48(\mathrm{~d}, J=6.3 \mathrm{~Hz}, 3 \mathrm{H}), 2.02$ $(\mathrm{s}, 1 \mathrm{H}, \mathrm{OH}), 3.81(\mathrm{~s}, 3 \mathrm{H}), 4.82-4.88(\mathrm{~m}, 1 \mathrm{H}), 6.79-6.82(\mathrm{~m}, 1 \mathrm{H}), 6.92-6.95(\mathrm{~m}, 2 \mathrm{H}), 7.25(\mathrm{t}, J=8.4$ $\mathrm{Hz}, 1 \mathrm{H}) ;{ }^{13} \mathrm{C} \mathrm{NMR}\left(75 \mathrm{MHz}, \mathrm{CDCl}_{3}\right) \quad \delta \quad 25.1,55.2,70.3,110.9,112.9,117.7,129.5,147.6,159.8$; GC analysis: CP-Chirasil-DEX CB column $(25 \mathrm{~m} \times 0.32 \mathrm{~mm})$, column temprature $=146{ }^{\circ} \mathrm{C}$, (isothemal), injector temprature $=250{ }^{\circ} \mathrm{C}$, detector temperature (FID) $=300$, carrier gas: $\mathrm{N}_{2}$, inlet 
pressure $=7 \mathrm{psi} ; \mathrm{t}_{\mathrm{R}}=11.0 \mathrm{~min}, \mathrm{t}_{\mathrm{S}}=11.7 \mathrm{~min}$

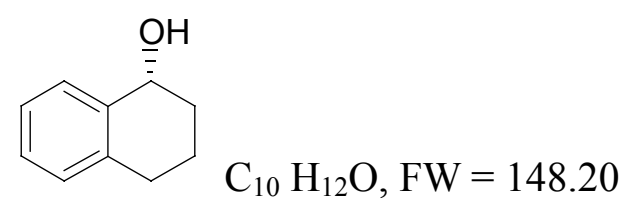

$[\alpha]_{\mathrm{D}}{ }^{28}-32\left(c 1.0, \mathrm{CHCl}_{3}\right) ;{ }^{1} \mathrm{H}$ NMR $\left(300 \mathrm{MHz}, \mathrm{CDCl}_{3},>99 \%\right.$ ee $) \quad \delta \quad 1.26-1.99(\mathrm{~m}, 4 \mathrm{H}), 2.71-2.84$ $(\mathrm{m}, 2 \mathrm{H}), 4.75(\mathrm{t}, J=4.2 \mathrm{~Hz}, 1 \mathrm{H}), 7.07-7.10(\mathrm{~m}, 1 \mathrm{H}), 7.16-7.23(\mathrm{~m}, 2 \mathrm{H}), 7.39-7.42(\mathrm{~m}, 1 \mathrm{H}) ;{ }^{13} \mathrm{C}$ NMR $\left(75 \mathrm{MHz}, \mathrm{CDCl}_{3}\right) \quad \delta \quad 18.8,29.2,32.2,68.1,126.1,127.5,128.6,128.9,137.0,138.8$; HPLC analysis: Chiralcel OB column, 2-propanol:hexane $=2.2: 97.8(1.0 \mathrm{~mL} / \mathrm{min}), \mathrm{t}_{\mathrm{R}}=11.3 \mathrm{~min}, \mathrm{t}_{\mathrm{S}}=16.6$ $\min$.

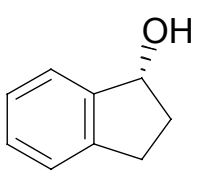

$$
\mathrm{C}_{9} \mathrm{H}_{10} \mathrm{O}, \mathrm{FW}=134.18
$$

Mp: $69-70{ }^{\circ} \mathrm{C} ;[\alpha]_{\mathrm{D}}{ }^{28}-31$ (c 1.3, $\mathrm{CHCl}_{3},>99 \%$ ee); ${ }^{1} \mathrm{H}$ NMR $\left(300 \mathrm{MHz}, \mathrm{CDCl}_{3}\right) \quad \delta 1.87(\mathrm{~s}, 1 \mathrm{H}$, $\mathrm{OH}), 1.90-1.99(\mathrm{~m}, 1 \mathrm{H}), 2.42-2.53(\mathrm{~m}, 1 \mathrm{H}), 2.76-2.86(\mathrm{~m}, 1 \mathrm{H}), 3.00-3.10(\mathrm{~m}, 1 \mathrm{H}), 5.23(\mathrm{~s}, 1 \mathrm{H})$, 7.22-7.25 (m, 3H), 7.39-7.42 (m, 1H); ${ }^{13} \mathrm{C}$ NMR (75 $\left.\mathrm{MHz}, \mathrm{CDCl}_{3}\right) \quad \delta \quad 29.8,35.9,76.4,124.2$, 124.9, 126.7, 128.3, 143.3, 145.0. HPLC analysis: Chiralcel OB column, 2-propanol:hexane = 5:95 $(1.0 \mathrm{~mL} / \mathrm{min}), \mathrm{t}_{\mathrm{R}}=8.4 \mathrm{~min}, \mathrm{t}_{\mathrm{S}}=12.1 \mathrm{~min}$.

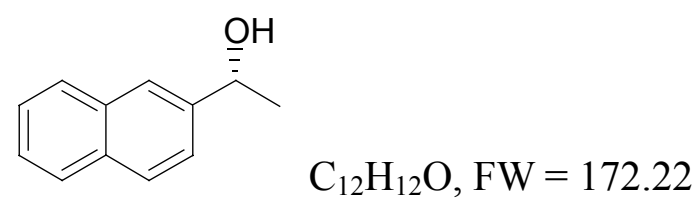

Mp: $68-70{ }^{\circ} \mathrm{C} ;[\alpha]_{\mathrm{D}}{ }^{28}+46\left(c 1.1, \mathrm{CHCl}_{3}, 95 \%\right.$ ee); ${ }^{1} \mathrm{H}$ NMR $\left(300 \mathrm{MHz}, \mathrm{CDCl}_{3}\right) \quad \delta$ 1.47-1.50 (m, $3 \mathrm{H}), 2.28(\mathrm{~s}, 1 \mathrm{H}, \mathrm{OH}), 5.13-5.19(\mathrm{~m}, 1 \mathrm{H}), 6.95-7.03(\mathrm{~m}, 1 \mathrm{H}), 7.09-7.26(\mathrm{~m}, 4 \mathrm{H}), 7.43-7.48(\mathrm{~m}, 2 \mathrm{H})$; ${ }^{13} \mathrm{C}$ NMR $\left(75 \mathrm{MHz}, \mathrm{CDCl}_{3}\right) \quad \delta \quad 23.9,64.4,115.1,115.3,124.3,126.6,128.6,128.7,130.9,132.5$, 158.0, 161.4; HPLC analysis: Chiralcel OJ column, 2-propanol:hexane $=7: 93(1.0 \mathrm{~mL} / \mathrm{min}), \mathrm{t}_{\mathrm{S}}=$ $18.6 \mathrm{~min}, \mathrm{t}_{\mathrm{R}}=23.8 \mathrm{~min}$. 


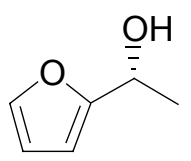

$\mathrm{C}_{6} \mathrm{H}_{8} \mathrm{O}_{2}, \mathrm{FW}=112.13$

$[\alpha]_{\mathrm{D}}{ }^{28}+9\left(c 1.4, \mathrm{CHCl}_{3}, 96 \%\right.$ ee); ${ }^{1} \mathrm{H}$ NMR $\left(300 \mathrm{MHz}, \mathrm{CDCl}_{3}\right) \quad \delta 1.54(\mathrm{~d}, J=6.6 \mathrm{~Hz}, 3 \mathrm{H})$, 4.85-4.91 (m, 1H), $6.23(\mathrm{~d}, J=3 \mathrm{~Hz}, 1 \mathrm{H}), 6.32-6.33(\mathrm{~m}, 1 \mathrm{H}), 7.37(\mathrm{~d}, J=1.2 \mathrm{~Hz}, 1 \mathrm{H}) ;{ }^{13} \mathrm{C} \mathrm{NMR}$ $\left(75 \mathrm{MHz}, \mathrm{CDCl}_{3}\right) \quad \delta$ 21.2, 63.6, 105.1, 110.1, 141.9, 157.6; GC analysis: CP-Chirasil-DEX CB column $\left(25 \mathrm{~m} \times 0.32 \mathrm{~mm}\right.$ ), column temprature: $60{ }^{\circ} \mathrm{C}$ hold $0.1 \mathrm{~min}$, ramp $3 / \mathrm{min}$ to $87{ }^{\circ} \mathrm{C}$ hold $3 \mathrm{~min}$, injector temprature $=250{ }^{\circ} \mathrm{C}$, detector temperature (FID) $=300$, carrier gas: $\mathrm{H}_{2}$, inlet pressure $=7$ $\mathrm{psi} ; \mathrm{t}_{\mathrm{R}}=9.8 \mathrm{~min}, \mathrm{t}_{\mathrm{S}}=10.4 \mathrm{~min}$.

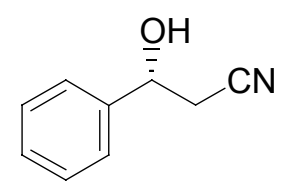

$$
\mathrm{C}_{9} \mathrm{H}_{9} \mathrm{NO}, \mathrm{FW}=147.17
$$

$[\alpha]_{\mathrm{D}}{ }^{28}+58\left(c 1.1, \mathrm{CHCl}_{3}, 97 \%\right.$ ee $) ;{ }^{1} \mathrm{H}$ NMR $\left(300 \mathrm{MHz}, \mathrm{CDCl}_{3}\right) \quad \delta \quad 2.69(\mathrm{~d}, J=6.0 \mathrm{~Hz}, 2 \mathrm{H}), 3.17$ (s, $1 \mathrm{H}, \mathrm{OH}), 4.96(\mathrm{t}, J=6.0 \mathrm{~Hz}, 1 \mathrm{H}), 7.30-7.40(\mathrm{~m}, 5 \mathrm{H}) ;{ }^{13} \mathrm{C} \mathrm{NMR}\left(75 \mathrm{MHz}, \mathrm{CDCl}_{3}\right) \quad \delta 27.8,69.8$, 117.4, 125.5, 128.6, 128.8, 141.0; HPLC analysis: Chiralcel OJ column, 2-propanol:hexane = 10:90 $(1.0 \mathrm{~mL} / \mathrm{min}), \mathrm{t}_{\mathrm{S}}=22.0 \mathrm{~min}, \mathrm{t}_{\mathrm{R}}=26.5 \mathrm{~min}$.

8. Reference: (1) Bennett, M. A.; Smith, A. K. J. Chem. Soc., Dalton Trans., 1974, 233. 